\title{
How healthy are the rhinoceros populations in the Hluhluwe-iMfolosi Park?
}

\author{
LP Fatti* \\ Received: 6 July 2012; Revised: 29 September 2012; Accepted: 5 December 2012 \\ Dedication to Emeritus Professor Theodor Stewart \\ To Theo Stewart, colleague, friend and inspiration.
}

\begin{abstract}
Arising from a study conducted in the Hluhluwe-iMfolosi Park by the author in the late nineties, a method is proposed for improving the estimate of the size of a wildlife population by combining data from current and past surveys. The method is based on a simple state space model which takes into account the (unknown) birth rate in the population and all known losses (mortalities and relocations) and gains (introductions) in the population between successive surveys, as well as the errors in the survey estimates. The method is applied to the White- and Black Rhinoceros populations in the Hluhluwe-iMfolosi Park and tentative conclusions are drawn on the health of these two populations.
\end{abstract}

Key words: State-space model, survey errors, birth rate, mortalities, rhinoceros.

\section{Introduction}

The Hluhluwe-iMfolosi Park in the Kwazulu-Natal Province is one of South Africa's major game reserves. It is particularly important for its population sizes of White and Black Rhinoceros ${ }^{1}$ (Ceratotherium simum and Diceros bicornis), being a key reserve in the campaign to save these species (Skinner and Smithers 1990). Because of the expense and unreliability of aerial surveys (Caughley 1974) sampling methods have been used in recent times to estimate the sizes of these two (and other) population sizes in the Park. The method used for White Rhino is based on observations taken along line transects passing through different sections of the Park, using a distance-based methodology to account for the decreasing detection rate with increasing distance between the observer and the animal (Buckland et al. 1993). In contrast, this method is inappropriate for Black Rhino with its shy habits, and instead a "mark-recapture" method ( Seber 1982) is used to estimate its

*(Fellow of the Operations Research Society of South Africa), School of Statistics and Actuarial Science, University of the Witwatersrand, Johannesburg, 2050, South Africa, email: paulfatti@gmail.com.

${ }^{1}$ Hereafter referred to as White and Black Rhino 
population size. When the author first became involved in 1997, data was available from eight surveys of the White Rhino which had been conducted between 1973 and 1996, and the park management was concerned about an apparent dramatic drop in population size between the last two surveys. The results of these surveys are displayed in Figure 1.

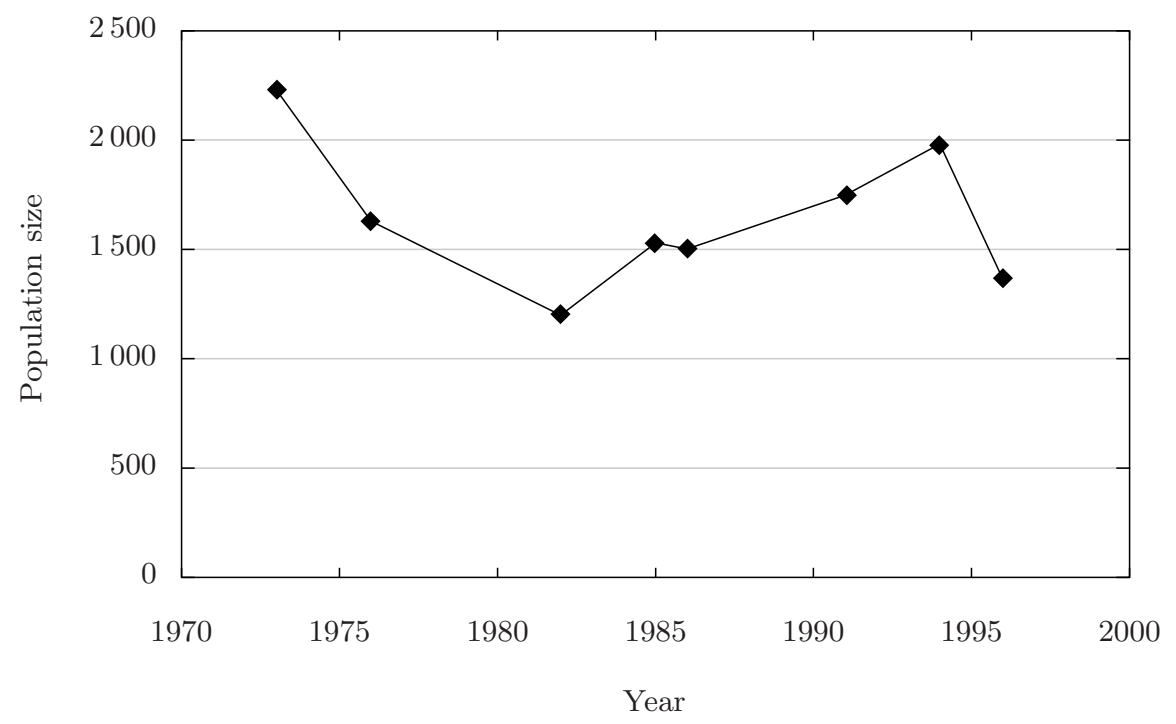

Figure 1: Survey estimates of the white rhino population size from 1973-1996.

From Figure 1 it would appear that the White Rhino population size had undergone some dramatic changes over the 24 years covered by these surveys. The apparent decline in numbers between the 1973 and 1982 surveys can partially be explained by the high numbers of relocations of Rhino (and other species) to other parks, because management needed to reduce stocking levels during the drought that affected the Park over much of this period. The steady growth over most of the subsequent period up to 1994 could be attributed to the recovery of the population after the drought, but there was no explanation for the sharp drop in numbers between the 1994 and 1996 surveys. A fear was expressed in some quarters that this drop could be (partially) explained by poaching that was not being officially reported.

However, for the scientists at the Park, the level of variability over time in the White Rhino population size, as indicated in Figure 1, is too great to be biologically realistic, even after accounting for the relocations and known mortalities of the animals. Their interpretation was that the survey estimates are subject to large errors and that the true underlying population size is far more stable than is suggested by the data. (The standard errors of the individual estimates, provided by the software (Buckland et al. 1993) which implements the distance based methodology, are indeed large.) The question therefore arises whether, by considering all eight surveys together, the individual estimates could not be adjusted to try to account for these survey errors. If this could be achieved, more realistic estimates of the actual population sizes at the times of the surveys and of the changes in between would be obtained, which in turn would allow the scientists and managers at the Park better insights into the natural and man-induced influences on the White Rhino population. 
In order to achieve this, we propose a simple model which combines the information from all the surveys, taking into account the net losses from the population sizes between successive surveys (through mortalities and removals, minus introductions), the (unknown) natural growth rate and the (unknown) error in the estimate at each survey. From this model we are able to obtain new estimates of the true population size at the times of each of the surveys, which incorporate the dynamics of the population size over time. The estimated natural growth rate also allows for short-term projections into the future.

\section{A model for the population size over time}

To combine the data from the eight surveys which were conducted over the 24-year period between 1973 and 1996, a model is required which will take into account the various known and unknown factors which have affected the population size over this time period. The known factors include the numbers of animals relocated from, and introduced to, the Park at different times between the surveys, as well as the numbers of mortalities in the Park, which are recorded from sightings by rangers. For large species such as rhino, it is unlikely that many, if any, dead specimens would be missed by the rangers during their regular patrols of the Park.

The unknown factors in the model include the natural rate of increase in the population size and, of course, the error in each of the survey estimates. The data for fitting the model comprise the estimate at each survey, the number of years between successive surveys and the known factors described above.

A simple discrete-time state-space model is proposed to describe the dynamics of the population size over time across successive surveys. The model comprises two components, the first being a deterministic process which describes the change of the (unknown) true population size from one survey to the next, taking into account the species' natural rate of increase as well as the relocations, introductions and mortalities which occurred in between. The second component describes the relationship between the survey estimates and the corresponding true population sizes by means of a simple stochastic error model.

Newman (1998) uses state-space modelling to describe the annual movements and mortalities of Pacific Coho Salmon populations over time. He employs stochastic models for both the unobservable fish movements and their mortalities, as well as for the process by which the population sizes are estimated. Parameter estimation and prediction are provided by Kalman filtering. Craig et al. (1997) use a hidden Markov model to describe how the Florida Manatee population sizes changes between successive surveys and the trend in its size over time, as well as the method by which these animals are counted. Bayesian inference, implemented via Markov Chain Monte Carlo simulation, is used to compute marginal posterior distributions for all model parameters and predictive distributions for future counts. Other models which have been used to analyse multiple animal surveys include the stochastic diffusion model, originally proposed by Dennis et al. (1991), which Nicholls et al. (1996) employ to estimate the probabilities of extinction of different herbivore species in the Kruger National Park.

The model presented here is somewhat simpler than most of the above models and employs 
few unknown parameters. The motivation is the principle of parsimony, particularly in view of the relatively small number of surveys which have been conducted in the HluhluweiMfolosi Park.

The following notation is used in this paper. Let

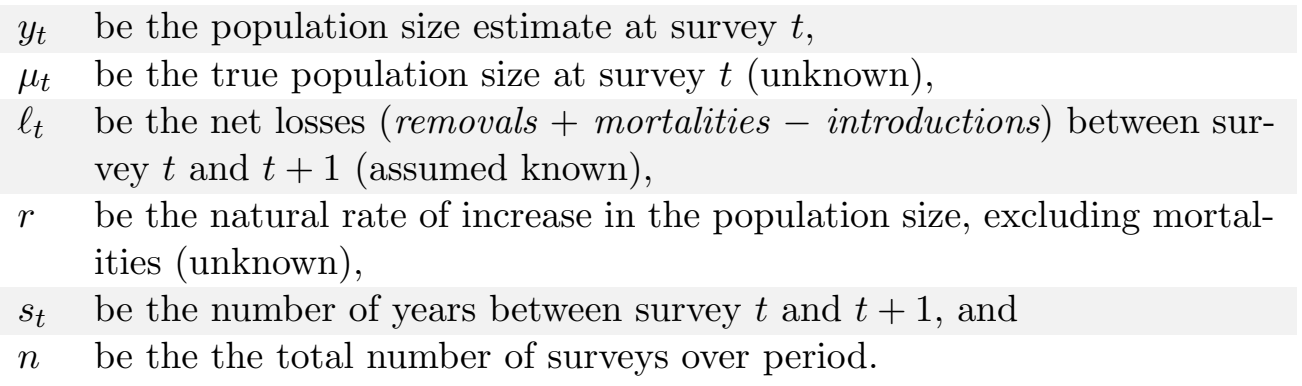

Assuming that the losses occur uniformly over time between successive surveys and that the natural rate of increase $r$ is constant over the whole period, the true population size at the time of survey $t+1$ is related to that at survey $t$ by

$$
\begin{aligned}
\mu_{t+1} & =\mu_{t} e^{r s_{t}}-\int_{0}^{s_{t}} \frac{\ell_{t}}{s_{t}} e^{r s_{t}} \mathrm{dt} \\
& =\mu_{t} e^{r s_{t}}-\ell_{t}\left(\frac{e^{r s_{t}}-1}{r s_{t}}\right),
\end{aligned}
$$

which takes into account the natural growth in the population size and the losses (including the growth which would have accrued from these animals had they not died or been removed). The assumption that the true population size at survey $t+1$ is deterministically related to that at $t$ is not strictly correct. However, in view of the generally large random observation error associated with the survey estimate, this assumption should be acceptable within the general accuracy of the model.

Assume that the estimate $y_{t}$ at survey $t$ is an unbiased estimator of the true population size but that there is an unknown, random observation error. The model for the estimate at survey $t$ therefore takes the simple form

$$
y_{t}=\mu_{t}+\varepsilon_{t},
$$

where $\varepsilon_{t}$ is a random error term which has an expected value zero (since $y_{t}$ is unbiased) and variance $\sigma^{2}$. Possibly $\varepsilon_{t}$ is Normally distributed, i.e. $\varepsilon_{t} \sim \mathrm{N}\left(0, \sigma^{2}\right)$, although this is not a necessary assumption. The assumption of a constant error variance $\sigma^{2}$ does not hold strictly for distance-based sampling estimates, as this variance is a function of the true population density (Buckland et al. 1993). However, unless the variation in the population density is considerable, this assumption should also be acceptable within the general accuracy of the approach.

Therefore, at survey $t+1$,

$$
\begin{aligned}
y_{t+1} & =\mu_{t+1}+\varepsilon_{t+1} \\
& =\mu_{t} e^{r s_{t}}-\ell_{t}\left(\frac{e^{r s_{t}}-1}{r s_{t}}\right)+\varepsilon_{t+1} \\
& =\left(y_{t}-\varepsilon_{t}\right) e^{r s_{t}}-\ell_{t}\left(\frac{e^{r s_{t}}-1}{r s_{t}}\right)+\varepsilon_{t+1},
\end{aligned}
$$


yielding the relationship

$$
\varepsilon_{t+1}=y_{t+1}-\left(y_{t}-\varepsilon_{t}\right) e^{r s_{t}}+\ell_{t}\left(\frac{e^{r s t}-1}{r s_{t}}\right)
$$

between the error at survey $t+1$ and that at the previous survey. Therefore the following algorithm is specified to fit the model.

(a) Start at $t=1$. Assign initial values to $\varepsilon_{1}$ and $r$.

(b) Now generate the error terms $\varepsilon_{2}, \ldots, \varepsilon_{n}$ successively as

$$
\varepsilon_{k}=y_{k}-\left(y_{k-1}-\varepsilon_{k-1}\right) e^{r s_{k-1}}+\ell_{k-1}\left(\frac{e^{r s_{k-1}}-1}{r s_{k-1}}\right) \quad \text { for } k=2, \ldots, n .
$$

(c) Estimate $\varepsilon_{1}$ and $r$ via least squares, i.e. find those values of $\varepsilon_{1}$ and $r$ that minimise $\sum_{t=1}^{n} \varepsilon_{t}^{2}$ using a nonlinear minimisation procedure. An estimate of the standard error of the survey estimates is taken as

$$
\hat{\sigma}=\sqrt{\frac{1}{n-2} \sum_{t=1}^{n} \varepsilon_{t}^{2}}
$$

taking into account the loss in degrees of freedom due to the estimation of the parameters $\varepsilon_{1}$ and $r$.

(d) Given the estimated $\hat{\varepsilon}_{1}$ and $\hat{r}$, successively compute the estimated true population size at each survey as

$$
\begin{aligned}
\hat{\mu}_{1} & =y_{1}-\hat{\varepsilon_{1}} \\
\hat{\mu}_{2} & =y_{2}-\hat{\varepsilon_{2}} \\
& =\left(y_{1}-\hat{\varepsilon_{1}}\right) e^{\hat{r} s_{1}}-\ell_{1}\left(\frac{e^{\hat{r} s_{1}}-1}{\hat{r} s_{1}}\right) .
\end{aligned}
$$

Therefore the true population size at survey $k$ is given by

$$
\hat{\mu}_{k}=\hat{\mu}_{k-1} e^{\hat{r} s_{k-1}}-\ell_{k-1}\left(\frac{e^{\hat{r} s_{k-1}}-1}{\hat{r} s_{k-1}}\right) \quad \text { for } k=2, \ldots, n .
$$

Remark 1: Experimentation with the nonlinear optimiser "Solver" add-in to the Microsoft Excel (Microsoft 2010) implementation of the algorithm described in the next section, has shown the least squares solution to be insensitive over a wide range of initial values for $\varepsilon_{1}$ and $r$. The initial values $\varepsilon_{1}=0$ and $r=0.1$ have generally been found to yield quick convergence.

Remark 2: It is usually the case that the mortality, relocation and introduction figures are recorded on an annual basis and not only as a total over the whole time interval between two successive surveys. If the annual net losses, $\ell_{t 1}, \ldots, \ell_{t s_{t}}$ which occurred between the $t^{\text {th }}$ and $t+1^{\text {th }}$ surveys are used in the algorithm, rather than their total, then the formula for the true population size at the time of survey $t+1$ becomes

$$
\mu_{t+1}=e^{r s_{t}}\left\{\mu_{t}-\left(\frac{e^{r}-1}{r}\right) \sum_{i=1}^{s_{t}}\left(\frac{\ell_{t i}}{e^{r i}}\right)\right\} .
$$

Formula (2) with estimated parameters then replaces (1). 


\section{Application to the Rhino populations in the Hluhluwe- iMfolosi Park}

The White Rhino estimates at each of the eight surveys, as well as the annual mortalities, relocations and introductions, which had occurred over the 24 years from 1973 to 1996, which was all the available data when the author first became involved in 1997, are given in the upper part of Table 1. Since the mortalities, relocations and introductions are recorded on a calendar year basis, whereas the surveys take place around the middle of the year, the former three sets of figures had to be interpolated for the model so as to correspond to the same time intervals as the surveys.

The algorithm described above has been implemented in an Microsoft Excel (Microsoft 2010) spread sheet and the "Solver" optimisation add-in is used for the minimisation step. The estimated true population sizes, growth rate and standard error of the surveys, derived from the algorithm, are also given in Table 1. Figure 2 superimposes the graph of the estimated true population sizes on that of the survey estimates at each of the eight surveys.

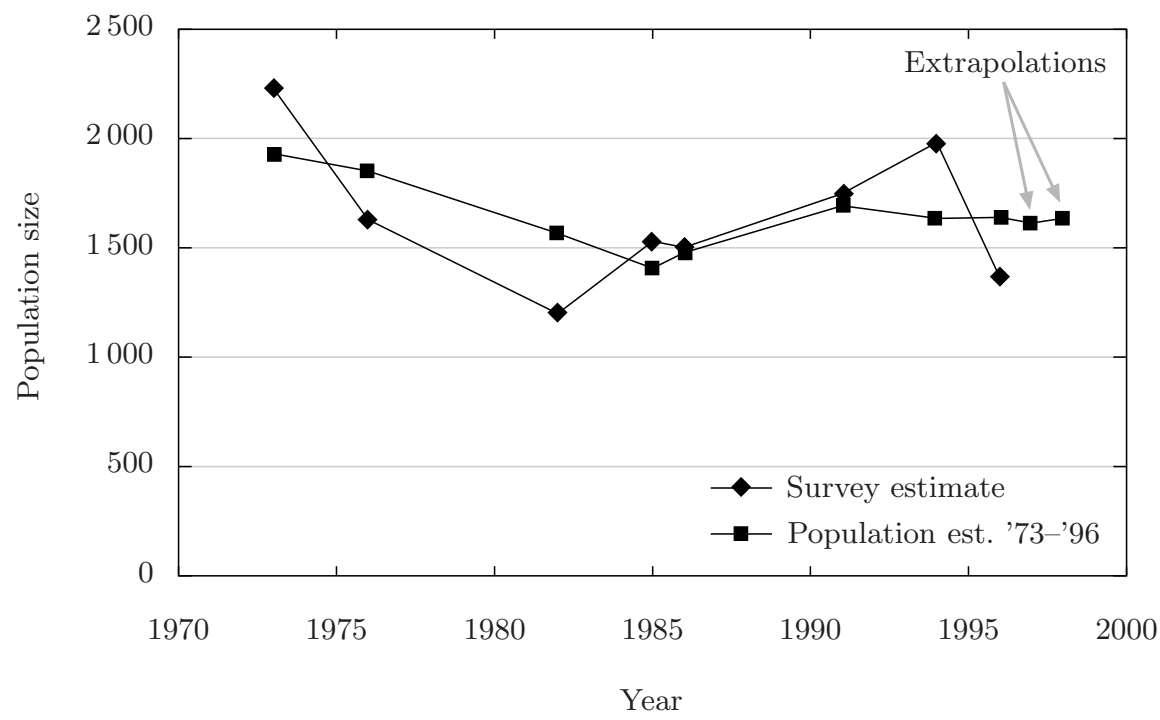

Figure 2: White rhino survey and population estimates from 1973-1996.

A useful piece of information deriving from the fitted model is the estimated natural growth rate per annum in the population size. The value estimated from the model is $r=0.076$ or $7.6 \%$, which falls well within the range of $6 \%$ to $8 \%$, considered to be realistic by the scientists at the Park, and this helped boost their confidence in the results of the model. The standard error of the survey estimates, obtained from the fitted model as described in Step 3 of the algorithm, is $\hat{\sigma}=286$.

The graph of the estimated true population sizes over time obtained from the model was considered to be far more realistic by the scientists than that obtained from the original survey estimates and gave them better insights into the true dynamics of the population over the 24 years. Interestingly, the sudden drop in numbers which occurred at the last survey, and which initiated the study reported here, does not have the largest observed 


\begin{tabular}{|c|c|c|c|c|c|c|c|}
\hline \multirow[b]{2}{*}{ Year } & \multirow[b]{2}{*}{ Survey } & \multirow[b]{2}{*}{ Mortalities } & \multirow[b]{2}{*}{ Poached } & \multirow[b]{2}{*}{ Removals } & \multirow[b]{2}{*}{ Introduced } & \multicolumn{2}{|c|}{ Population estimates } \\
\hline & & & & & & 1973-1996 & $1973-2008$ \\
\hline 1973 & 2230 & 19 & 0 & 269 & 0 & 1932 & 1980 \\
\hline 1974 & & 18 & 0 & 196 & 0 & & \\
\hline 1975 & & 15 & 0 & 100 & 0 & & \\
\hline 1976 & 1629 & 10 & 0 & 44 & 0 & 1852 & 1893 \\
\hline 1977 & & 14 & 0 & 33 & 0 & & \\
\hline 1978 & & 8 & 0 & 55 & 0 & & \\
\hline 1979 & & 22 & 0 & 154 & 0 & & \\
\hline 1980 & & 47 & 0 & 438 & 0 & & \\
\hline 1981 & & 14 & 0 & 156 & 0 & & \\
\hline 1982 & 1199 & 37 & 0 & 334 & 0 & 1567 & 1587 \\
\hline 1983 & & 42 & 0 & 131 & 0 & & \\
\hline 1984 & & 35 & 0 & 81 & 0 & & \\
\hline 1985 & 1530 & 20 & 0 & 3 & 0 & 1406 & 1416 \\
\hline 1986 & 1502 & 8 & 0 & 45 & 0 & 1478 & 1484 \\
\hline 1987 & & 18 & 0 & 19 & 0 & & \\
\hline 1988 & & 24 & 0 & 27 & 0 & & \\
\hline 1989 & & 24 & 0 & 83 & 0 & & \\
\hline 1990 & & 23 & 0 & 81 & 0 & & \\
\hline 1991 & 1748 & 31 & 0 & 128 & 0 & 1693 & 1671 \\
\hline 1992 & & 25 & 0 & 60 & 0 & & \\
\hline 1993 & & 47 & 0 & 158 & 0 & & \\
\hline 1994 & 1982 & 26 & 0 & 119 & 0 & 1636 & 1591 \\
\hline 1995 & & 25 & 0 & 47 & 0 & & \\
\hline 1996 & 1364 & 36 & 0 & 179 & 12 & 1640 & 1578 \\
\hline 1997 & & 34 & 0 & 59 & 0 & & \\
\hline 1998 & 1542 & 33 & 0 & 46 & 0 & & 1572 \\
\hline 1999 & & 51 & 0 & 23 & 0 & & \\
\hline 2000 & 1687 & 43 & 4 & 29 & 0 & & 1657 \\
\hline 2001 & & 28 & 1 & 52 & 0 & & \\
\hline 2002 & 1802 & 24 & 0 & 22 & 0 & & 1764 \\
\hline 2003 & & 29 & 1 & 98 & 0 & & \\
\hline 2004 & 1918 & 27 & 0 & 0 & 0 & & 1864 \\
\hline 2005 & & 27 & 18 & 53 & 0 & & \\
\hline 2006 & & 28 & 2 & 40 & 0 & & \\
\hline 2007 & & 14 & 0 & 79 & 0 & & \\
\hline \multirow[t]{3}{*}{2008} & 2038 & 25 & 10 & 37 & 0 & & 2140 \\
\hline & & & \multirow{2}{*}{\multicolumn{3}{|c|}{$\begin{array}{l}\text { Estimated population growth rate } \\
\text { Standard error of survey estimates }\end{array}$}} & $7.6 \%$ & $7.3 \%$ \\
\hline & & & & & & 286 & 217 \\
\hline
\end{tabular}

Table 1: White rhino survey data and population size estimates during the period 1973-2008.

error associated with it. Instead, it is the 1982 survey figure that is furthest from the estimated true population size. This figure is considered to be too low and instead the model gives a far more gradual decline in numbers than is suggested by the original survey estimates. Also, instead of the apparent large drop in the population size between the 1994 and 1996 surveys, the model estimates a slight increase in numbers.

Figures for the Black Rhino population size in the Park, obtained from "mark-recapture" surveys in each of the seven years from 1990 to 1996, are given in the upper part of Table 2. (Application of the mark-recapture methodology in the Hluhluwe-iMfolosi Park requires rangers to record whenever they see a Black Rhino and to note from the animal's 
markings whether or not it is one of the known individuals in the Park.) Figure 3 displays the estimated true Black Rhino population size from the model, superimposed on the corresponding survey estimates from each of these years.

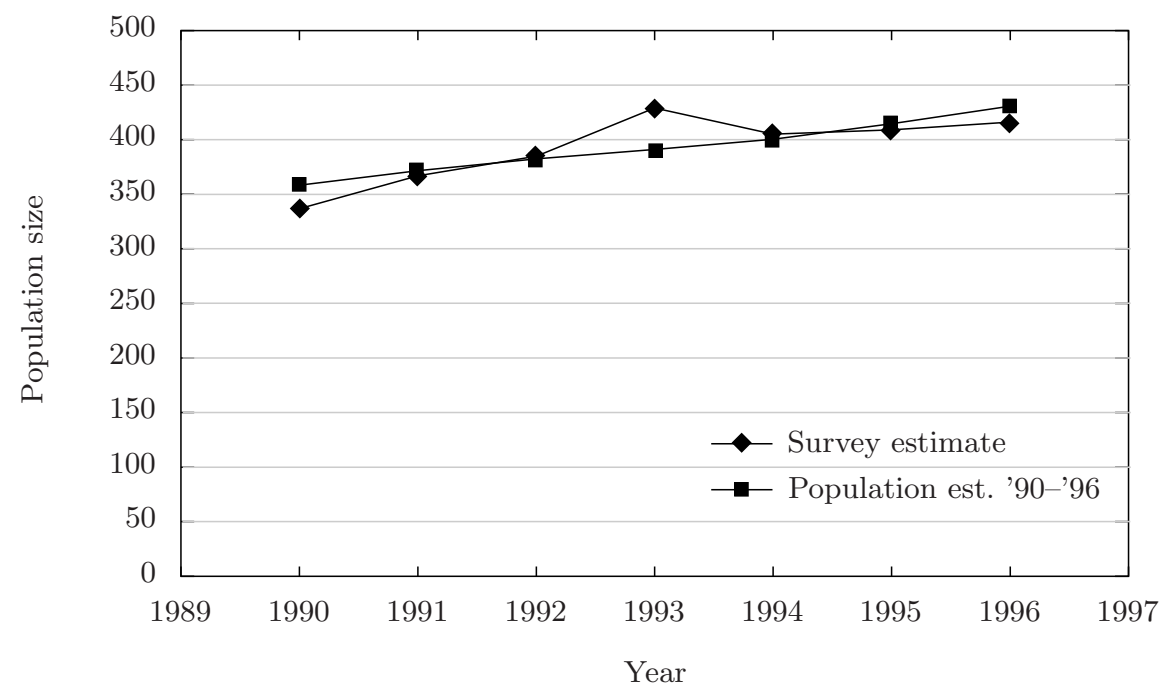

Figure 3: Black rhino surveys and population estimates from 1990-1996.

The estimated growth rate obtained from the fitted model is $8.6 \%$, which is a little higher than the scientists were expecting, but is not unrealistic. The standard error of the survey estimates is $\hat{\sigma}=21$ and it is evident that the variability of the estimates of the Black Rhino population size, obtained from the "mark-recapture" method, is much smaller than that of the White Rhino population size, obtained using the distance-based method. Nevertheless, the model provides a much smoother curve for the Black Rhino population size than do the survey estimates, showing it increasing steadily over time. In particular, it clearly identifies the 1993 survey estimate as being somewhat too high.

A shortcoming of the population size estimates provided by the model is that their standard errors are unknown. An estimate of the standard error may be obtained through application of the Jackknife method (Quennouille, 1956). Essentially, this entails dropping one of the surveys from sample, fitting the model on the remaining $n-1$ survey estimates and using this model to estimate the population size $\mu_{t}$ in the particular year of interest. This process is repeated over all surveys, resulting in $n$ estimates, $\hat{\mu}_{t(1)}, \ldots, \hat{\mu}_{t(n)}$ of $\mu_{t}$. Using the theory of the Jackknife method, the standard error of the population size estimate $\hat{\mu}_{t}$ may then be estimated as

$$
\hat{\sigma}_{\hat{\mu}_{t}}=\sqrt{\left(\frac{n-1}{n}\right) \sum_{i=1}^{n}\left(\hat{\mu}_{t(i)}-\hat{\mu}_{t(\cdot)}\right)^{2}},
$$

where $\hat{\mu}_{t(\cdot)}$ is the average of the $\hat{\mu}_{t(i)}$ for $i=1, \ldots, n$ (Efron, 1982). This is a slightly conservative estimate of the standard error, since it refers to a situation where there have been $n-1$ (not $n$ ) surveys.

Applying this method to the estimated White Rhino population size in 1996 yields a value of 80 for the standard error of $\hat{\mu}_{1996}$, which is less than $30 \%$ of the estimated standard error 


\begin{tabular}{cccccccc}
\hline & & & & & Population estimates \\
Year & Survey & Mortalities & Poached & Removals & Introduced & $1990-1996$ & $1990-2008$ \\
\hline 1990 & 337 & 11 & 0 & 8 & 0 & 358 & 358 \\
1991 & 367 & 10 & 0 & 7 & 0 & 372 & 372 \\
1992 & 385 & 16 & 0 & 10 & 0 & 382 & 382 \\
1993 & 429 & 22 & 0 & 2 & 0 & 391 & 391 \\
1994 & 405 & 9 & 0 & 16 & 0 & 400 & 400 \\
1995 & 409 & 3 & 0 & 14 & 0 & 414 & 414 \\
1996 & 416 & 8 & 0 & 17 & 2 & 431 & 430 \\
1997 & 409 & 10 & 0 & 29 & 0 & & 407 \\
1998 & 376 & 8 & 0 & 18 & 1 & & 382 \\
1999 & 363 & 16 & 0 & 14 & 0 & & 361 \\
2000 & 325 & 11 & 0 & 27 & 0 & & 334 \\
2001 & 303 & 16 & 0 & 7 & 0 & & 296 \\
2002 & 293 & 12 & 0 & 1 & 0 & & 285 \\
2003 & 301 & 12 & 0 & 9 & 1 & & 272 \\
2004 & 288 & 8 & 0 & 10 & 1 & & 259 \\
2005 & 260 & 4 & 0 & 15 & 0 & & 236 \\
2006 & 246 & 4 & 0 & 12 & 0 & & 226 \\
2007 & 228 & 2 & 0 & 12 & 0 & & $8.6 \%$ \\
2008 & 218 & 6 & 0 & 6 & 0 & & 13 \\
\hline \multicolumn{7}{c}{ Estimated population growth rate from 1990-1996 } \\
\hline \multicolumn{7}{c}{ Estimated population growth rate from 1997-2008 } \\
\hline
\end{tabular}

Table 2: $\quad$ Black rhino survey data and population size estimates during the period 1990-2008.

of the corresponding survey estimate. The corresponding figure for the standard error of the Black Rhino population size is 16 , showing a more modest reduction from that of 21 for the survey estimates.

Over and above its use in providing more realistic population size estimates than those from the original surveys, the model can also be used for short-term forecasting. For any assumed number of relocations, mortalities and introductions over a given time it is an easy matter to extrapolate the fitted model forward from the last population size estimate $\hat{\mu}_{n}$. Figure 2 also shows the extrapolated White Rhino population size for two years ahead of the 1996 survey, assuming a net value of 100 for the relocations, mortalities and introductions in each of these two years. The use of such extrapolations as a management tool to test the effects of different relocation policies on the population size is evident.

The author has now obtained more recent data on both the White and Black Rhino population sizes up to 2008 from the Park authorities. Applying the model to the full sets of data gives insight on the population size dynamics over the whole period from 1973 for the White Rhino and from 1990 for the Black Rhino, and allows a comparison between the earlier dynamics of these population sizes with those of more recent years. This data appears in the lower parts of Tables 1 and 2 for the White and Black Rhino, respectively.

There have been a further five White Rhino surveys from 1996 to 2008, giving a total of 13 since 1973. Figure 4 presents the figures from each of the 13 surveys together with the corresponding population size estimates obtained from the model fitted to all the data since 1973. The fit of the model to the data over the last five surveys is seen to be very 
close, bringing the estimated standard error down to 217 from its earlier value of 286 obtained from the model fitted to the first eight surveys. The estimated natural growth rate in the population size over the whole period is $7.3 \%$, which compares well with the figure of $7.6 \%$ for the period up to 1996 . The generally good fit of the model over the whole period shows that the White Rhino population size has maintained a healthy profile, taking into account the removals and mortalities (36 of which have been due to poaching between 2000 and 2008) and the 12 which were introduced into the Park in 1996.

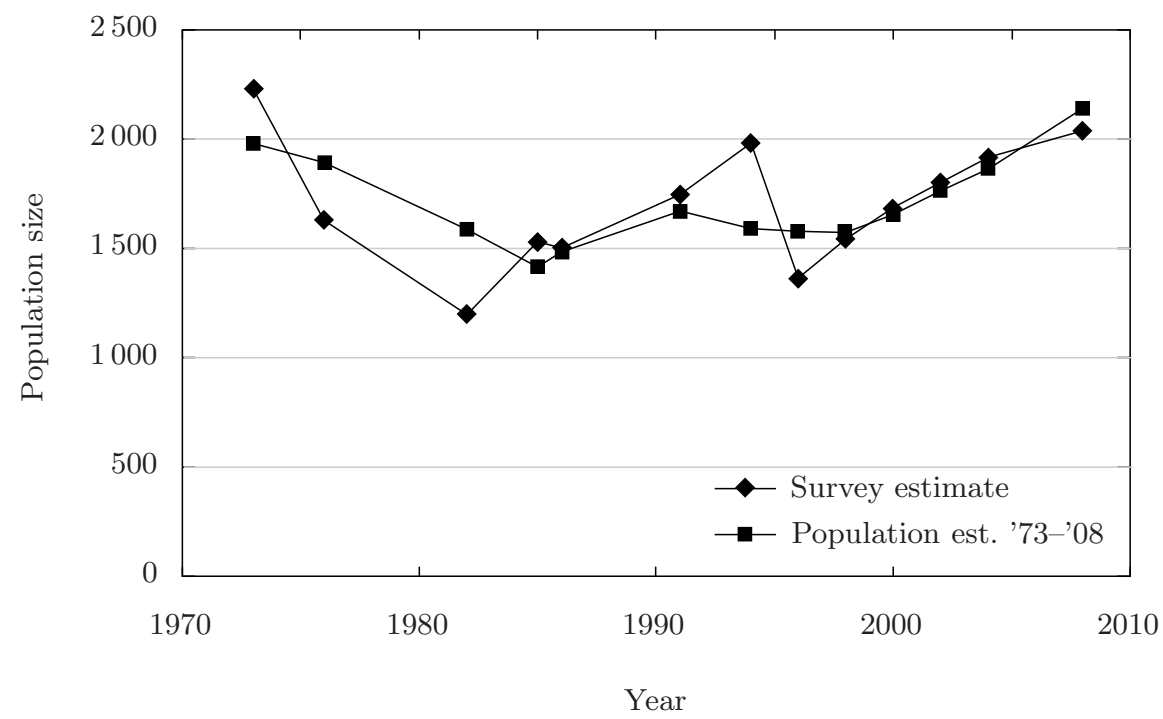

Figure 4: White rhino surveys and population size estimates from 1973-2008.

The picture with the Black Rhino is dramatically different. There have been "markrecapture" estimates for every year from 1990 to 2008, i.e. 19 in all, of which 12 have been after 1996. Figure 5 presents these 19 estimates as well as the corresponding population size estimates obtained from the model fitted to all the data.

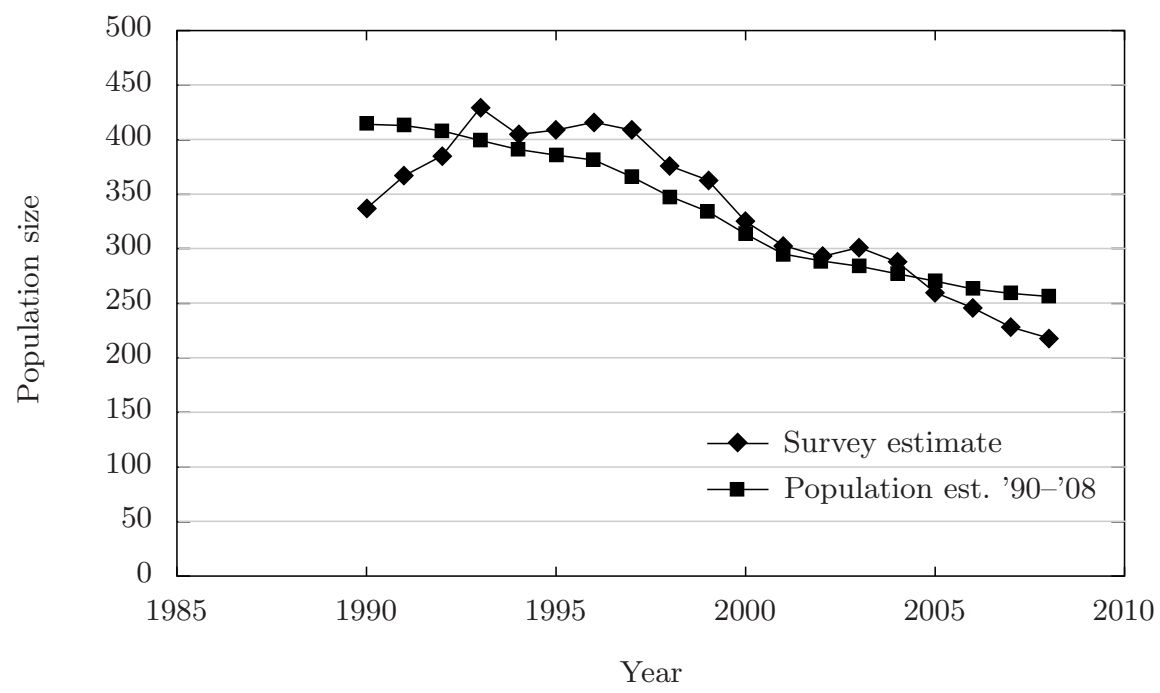

Figure 5: Black rhino surveys and population size estimates from 1990-2008. 
The fit of this model is far worse than that of the model fitted to the 7 surveys from 1990 to 1996 . Whereas the latter model shows a steady population size growth at an estimated rate of $8.6 \%$, the survey estimates go into decline after that, pulling down the population size estimates and producing an estimated population size growth rate over the whole period of only $4.1 \%$. The estimated standard error of the survey estimates is 33 , considerably higher than that of 21 obtained from the model fitted to the first 7 surveys, indicating that the model fits poorly to the data over the whole period.

It seems clear that the model's assumption of a constant growth rate over the whole period is a reason for its poor fit. In view of the apparent drop in the growth rate after 1996 the model was therefore relaxed by assuming separate growth rates for the period up to 1996 and for the period after that. Fitting the relaxed model to the survey data for the whole period shows a dramatic drop in the estimated population size growth rate from $8.6 \%$ in the first period to $1.8 \%$ in the second period. The improved fit of this model is evident from Figure 6 and the standard error of only 13 confirms that it gives a much better description of the dynamics of the Black Rhino population size between 1990 and 2008.

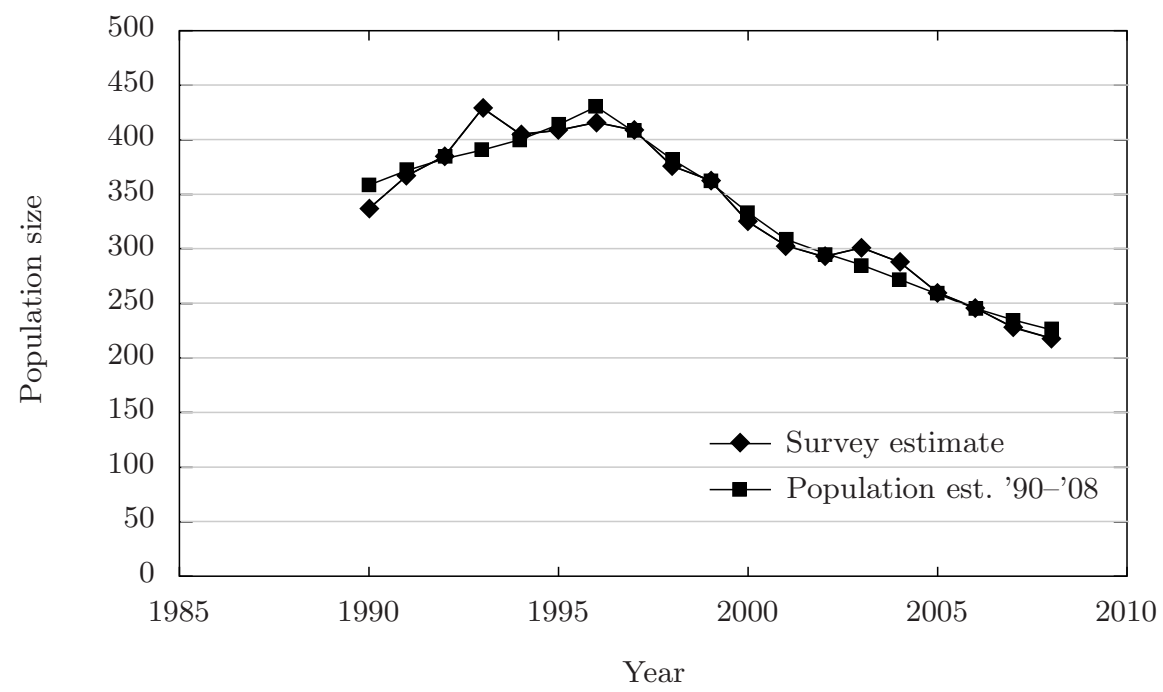

Figure 6: Black rhino surveys and population size estimates from 1990-2008, two growth rates.

\section{Discussion}

In this paper a model has been introduced for analysing the dynamics of population sizes of large animal species in a game park from successive survey estimates (or "censuses"), including the counts of relocations, mortalities (including those from poaching) and introductions between successive surveys. The model has a simple structure with few unknown parameters and can therefore be applied to population sizes which have had relatively few surveys. One of the unknown parameters that is estimated when fitting the model is the natural annual rate of increase in the population size (excluding mortalities), and an estimate of the standard error of the survey estimates may also be obtained from the 
model results. The model may be applied to populations for which surveys are undertaken at irregularly spaced intervals. The algorithm for fitting the model can be implemented in a spread sheet which includes a general optimisation tool.

The model has been applied to the White and Black Rhino populations in the HluhluweiMfolosi Park, initially in 1997, after the Park experienced an apparent sharp decline in its White Rhino population size in the 1996 survey. The model, which was developed to analyse this situation, showed that the sharp decline in numbers at the last survey could be explained by random error alone. The model fitted to the Black Rhino survey results showed the population size to be increasing at a steady rate of $8.6 \%$ per annum.

The model has now been applied to the surveys of the White and Black Rhino population sizes in the Park including those that have taken place in the subsequent twelve years up to 2008. The model fitted to the 13 White Rhino surveys that had taken place since 1973 (8 up to 1996 and 5 subsequently) was consistent with the model fitted to the first 8 surveys alone and showed that the population size was stable with a natural annual rate of increase of $7.3 \%$ (compared to $7.6 \%$ for the model fitted to the data up to 1996).

The situation with the Black Rhino population size is dramatically different and shows that after the steady increase up to 1996 the numbers decrease sharply, continuing until 2008. The model provided a poor fit to the survey data and it was evident that the assumption of a constant natural rate of increase in the population size did not hold. So instead, the model assumptions were relaxed slightly by using two rates of increase, the first holding until 1996 and the second applying after that. The model applied under this relaxed assumption fitted the survey data very well over the whole period from 1990 to 2008 , and showed that while the estimated natural rate of increase up to 1996 was still $8.6 \%$ per annum, after 1996 it had dropped down to $1.8 \%$.

It is interesting to speculate what the reason is for this sharp drop in the natural rate of increase in the Black Rhino population size. As is evident from the data in Tables 1 and 2, while 36 White Rhino were reported as having been poached between 2000 and 2008, no poaching of Black Rhino was reported over the whole period from 1990 to 2008. Unreported poaching of Black Rhino (or any other unreported mortalities or removals) after 1996 would clearly have had an effect on the model estimates. (However, the scientists at the Park dismiss this possibility, as poachers only remove the rhino horn, leaving the carcass behind which will be discovered by the Park rangers.) Another reason could be the reduction in woody terrain in the Park, favoured for browsing by the Black Rhino, as a result of the large White Rhino population size which favours open grassland for grazing. The fire management policy of the Park could have been aimed at increasing the grassland, resulting in a reduction of the woody terrain.

The current thinking of the scientists at the Park is that the earlier Black Rhino estimates were inflated as result of shortcomings in the implementation of the "mark-recapture" survey method, which have subsequently been resolved. They are currently re-visiting the data from the earlier surveys and will be producing revised estimates for these years. The model described in this paper will then be applied to these revised estimates, and hopefully the picture that emerges will be more hopeful for the Black Rhino population size. 
An important outcome of the study is that it confirms the usefulness of the model developed in Section 2. For the White Rhino data the model produces realistic population size estimates, accounting for the mortalities, removals and introductions, and provides sensible estimates of the natural growth rate. The model can also produce short-term forecasts of the population size, using assumptions about the future numbers of removals, mortalities and introductions, which has obvious application as a management tool.

For the Black Rhino, the initially poor fit of the model is resolved by introducing a second growth rate parameter, which dramatically improves the fit and also points to the sharp drop in the growth rate between the period up to 1996 and that from 1997 onwards. This drop cannot be ascribed to the known mortalities and removals, since the model takes account of them, and therefore points to possible problems, either with the Black Rhino population size itself, or with the earlier application of the "mark-recapture" survey method, as discussed above.

\section{Acknowledgements}

The author would like to express his gratitude Dr David Balfour, former Park Ecologist, for introducing him to the problem in 1997; Dr Dave Druce, current Park Ecologist, for discussion of the findings regarding the Black Rhino; Mr Stuart Corrigal, Masters student in 1997, for initially implementing the model in MS Excel (this model is still being used by the scientists in the Park); and Ezemvelo KZN Wildlife for generously providing the data from the Rhino surveys.

\section{References}

[1] Buckland ST, Anderson DR, Burnham KP \& LaAke JL, 1993, Distance sampling: Estimating abundance of biological populations, Chapman \& Hall, London.

[2] Caughley G, 1974, Bias in aerial survey, Journal of Wildlife Management, 38(4), pp. 921-933.

[3] Craig BA, Newton MA, Garrott RA, Reynolds III JE \& Wilcox JR, 1997, Analysis of aerial survey data on Florida manatee using markov chain monte carlo, Biometrics, 53(2), pp. 524-541.

[4] Dennis B, Munholland PL \& Scott JM, 1991, Estimation of growth and extinction parameters for endangered species, Ecological Monographs, 61(2), pp. 115-143.

[5] Efron B, 1982, The jackknife, the bootstrap and other resampling plans, SIAM, Philadelphia (PA).

[6] Microsoft, 2010, Excel, [Online], [Cited December 12 $\left.{ }^{\text {th }} 2012\right]$, Available from http://office. microsoft.com/en-us/excel/.

[7] Newman KB, 1998, State-space modeling of animal movement and mortality with application to salmon, Biometrics, 54(4), pp. 1290-1314.

[8] Nicholls AO, Viljoen PC, Knight MH \& van JaARsveld AS, 1996, Evaluating population persistence of censused and unmanaged herbivore populations from the Kruger National Park, South Africa, Biological Conservation, 76(1), pp. 57-67.

[9] Quenoullle M, 1956, Notes on bias in estimation, Biometrika, 43(3,4), pp. 353-360.

[10] SEBER GAF, 1982, The estimation of animal abundance and related parameters, $2^{\text {nd }}$ Edition, Charles Griffen, London.

[11] Skinner JD \& Smithers RHN, 1990, Mammals in the Southern African Subregion, University of Pretoria, Pretoria. 